\title{
Dispersion of SH and Love Waves
}

\author{
Sergey V. Kuznetsov* \\ Institute for Problems in Mechanics, Prosp. Vernadskogo, Moscow, Russia \\ *Corresponding author: kuzn-sergey@yandex.ru
}

Received June 09, 2014; Revised June 20, 2014; Accepted October 15, 2014

\begin{abstract}
A mathematical model for analyzing both Love waves and horizontally polarized shear surface waves (SH-waves) propagating in stratified media with monoclinic symmetry is worked out. Analytic and numerical solutions for SH and Love waves obtained by applying the Modified Transfer Matrix (MTM) method and a special complex formalism, are presented. Displacement fields, specific energy, phase, ray, and group velocities, and dispersion curves for $\mathrm{SH}$ and Love waves are compared and analyzed. Plates with different types of boundary conditions imposed on the outer surfaces are considered. Behavior of the leakage Love waves and anomalous SHwaves is discussed.
\end{abstract}

Keywords: SH-wave, shear wave, surface wave, Love wave, dispersion, laminated plate

Cite This Article: Sergey V. Kuznetsov, "Dispersion of SH and Love Waves." International Journal of Physics, vol. 2, no. 5 (2014): 170-180. doi: 10.12691/ijp-2-5-7.

\section{Introduction}

Horizontally polarized shear surface waves (SH-waves) propagating in multilayered plates resemble Love waves [1] in polarization, but differ in absence of a contacting half-space (substrate), and, hence excluding necessity to impose Sommerfeld's emission condition:

$$
\mathbf{u}(\mathbf{x}, t)=O\left(\left|x^{\prime}\right|^{-1}\right), \quad\left|x^{\prime}\right| \rightarrow \infty
$$

where $\mathbf{u}$ is the displacement field in the substrate; $x^{\prime} \equiv \mathbf{v} \cdot \mathbf{x}$ is the coordinate along depth of the substrate, and $v$ is the unit normal to the plane boundary of the substrate; see Figure 1.

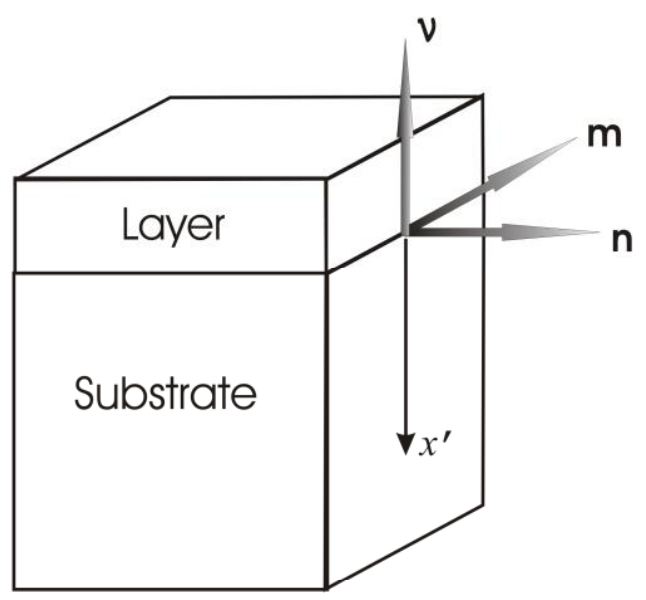

Figure 1. Base vectors

As will be shown later, absence of condition (1.1) results in a completely different behavior of the $\mathrm{SH}$-waves in layered plates comparing to Love waves. For example, it is known an existence inequality [2] for a genuine Love wave propagating in an isotropic traction-free layer contacting with isotropic substrate:

$$
\left(c_{\mathbf{n m}}^{T}\right)_{\text {layer }}<\left(c_{\mathbf{n m}}^{T}\right)_{\text {substrate }}
$$

where $c_{\mathbf{n m}}^{T}$ denotes speed of the corresponding shear bulk wave propagating in $\mathbf{n}$, and polarized in $\mathbf{m}$ direction. Violating inequality (1.2) prevents Love waves to exist. As will be shown later, the $\mathrm{SH}$-waves in two-layered plates exist at any physically admissible properties of homogeneous layers and at traction-free, clamped, or mixed boundary conditions (one outer surface is traction free, and another is clamped).

The main method used for constructing analytic and numerical solutions for $\mathrm{SH}$ and Love waves, is based on a combination of a complex formalism $[3,4]$ and the modified transfer matrix (MTM) method [5,6]. The latter method along with the multiprecision computations allowed us to avoid numerical instability of the solution arising at layers of large thickness and high frequencies of the waves. This is known as the "large $h \omega$-problem', where $h$ is the layer thickness and $\omega$ is frequency; see $[7,8,9,10]$. We should also mention that herein we do not consider the exceptional waves corresponding to appearing Jordan blocks in the Christoffel equation; see [5].

Other methods for analyzing propagation of $\mathrm{SH}$ and Love waves in connection to geophysical and geotechnical applications, are presented in recent publications $[11,12,13,14]$.

\section{Basic Notations}

All the regarded layers and a substrate are assumed to be homogeneous, anisotropic and linearly hyperelastic. Equations of motion for homogeneous anisotropic elastic medium can be written in the form: 


$$
\mathbf{A}\left(\partial_{x}, \partial_{t}\right) \mathbf{u} \equiv \operatorname{div}_{x} \mathbf{C} \cdot \nabla_{x} \mathbf{u}-\rho \ddot{\mathbf{u}}=0
$$

where $\rho$ is the material density, and $\mathbf{C}$ is the elasticity tensor assumed to be positive definite:

$$
\begin{aligned}
& \underset{i, j, m, n}{\forall \mathbf{A}} A_{i j} C^{i j m n} A_{m n}>0 \\
& \equiv \sum^{\mathbf{A} \in \operatorname{sym}\left(R^{3} \otimes R^{3}\right), \quad \mathbf{A} \neq 0} \quad(\mathbf{A} \cdot \mathbf{C} \cdot \cdot \mathbf{A})
\end{aligned}
$$

REMARK 2.1. a) The other assumption concerns symmetry of the elasticity tensor. It will be assumed that all the regarded materials possess planes of elastic symmetry coinciding with the sagittal plane $\mathbf{m} \cdot \mathbf{x}=0$, where vector $\mathbf{m}$ is the polarization vector of the SH-wave. This is achieved by the elasticity tensor belonging to the monoclinic system, and the latter is equivalent to vanishing all of the decomposable components of the tensor $\mathbf{C}$ having odd number of entries of the vector $\mathbf{m}$ in the orthogonal basis in $R^{3}$ generated by the vector $\mathbf{m}$ and any two orthogonal vectors belonging to the sagittal plane.

b) It will be shown later that assuming monoclinic symmetry provides a sufficient condition for the surface tractions acting on any plane $\boldsymbol{v} \cdot \mathbf{x}=$ const to be collinear with vector $\mathbf{m}$.

Following [5], we will seek a horizontally polarized shear wave in a layer or substrate in the form:

$$
\mathbf{u}(\mathbf{x})=\mathbf{m} f\left(i r x^{\prime}\right) e^{i r(\mathbf{n} \cdot \mathbf{x}-c t)},
$$

where coordinate $x^{\prime}=\mathbf{v} \cdot \mathbf{x}$ is as defined in (1.1); $f$ is the unknown scalar complex-valued function; the exponential multiplier $\operatorname{ir}(\mathbf{n} \cdot \boldsymbol{v}-c t)$ in (2.3) corresponds to propagation of the plane wave front along direction $\mathbf{n}$ with the phase speed $c ; r$ is the wave number.

REMARK 2.2. The displacement field defined by (2.3) is generally complex. In reality, either real or imaginary part of the right-hand side of (2.3) represents physical displacement field that will be implicitly assumed in the subsequent analysis. However, retaining complex expressions for the displacement field, will allow us to describe situations with the phase shift in a more convenient manner.

Substituting representation (2.3) into Eq. (2.1) and taking into account Remark 2.1.a, yields the following differential equation:

$$
\left(\begin{array}{l}
(\mathbf{m} \otimes \boldsymbol{v} \cdot \mathbf{C} \cdot \cdot \boldsymbol{v} \otimes \mathbf{m}) f_{x^{\prime}}^{\prime \prime} \\
+2(\mathbf{m} \cdot \operatorname{sym}(\boldsymbol{v} \cdot \mathbf{C} \cdot \mathbf{n}) \cdot \mathbf{m}) f_{x^{\prime}}^{\prime} \\
+\left(\mathbf{m} \otimes \mathbf{n} \cdot \mathbf{C} \cdot \mathbf{n} \otimes \mathbf{m}-\rho c^{2}\right) f
\end{array}\right)=0
$$

Characteristic equation for the differential equation (2.4), known also as the Christoffel equation, has the form:

$$
\begin{aligned}
& (\mathbf{m} \otimes \boldsymbol{v} \cdot \mathbf{C} \cdot \cdot \boldsymbol{v} \otimes \mathbf{m}) \gamma^{2} \\
& +2(\mathbf{m} \cdot \operatorname{sym}(\boldsymbol{v} \cdot \mathbf{C} \cdot \mathbf{n}) \cdot \mathbf{m}) \gamma \\
& +\left(\mathbf{m} \otimes \mathbf{n} \cdot \mathbf{C} \cdot \mathbf{n} \otimes \mathbf{m}-\rho c^{2}\right)=0
\end{aligned}
$$

Left-hand side of Eq. (2.5) represents a polynomial of degree 2 with respect to the Christoffel parameter $\gamma$. Thus, for the monoclinic elastic symmetry only two partial waves form the regarded SH-wave in a layer, and one partial wave with $\operatorname{Im}(\gamma)<0$ forms Love wave in the substrate; see [5].

The following lemma flows out from solving the Cauchy problem for Eq. (2.4):

LEMMA 2.1. A necessary and sufficient condition for the real-analytic solution of Eq.(2.4), to be a non-zero function, is a simultaneous non-vanishing $f$ and its first derivative at some $x^{\prime}$.

REMARK 2.3. a) For an orthotropic medium and the SHwave propagating in a direction of the principle elasticity, Eq.(2.5) is simplified:

$$
\begin{aligned}
& (\mathbf{m} \otimes \mathbf{v} \cdot \mathbf{C} \cdot \cdot \mathbf{v} \otimes \mathbf{m}) \gamma^{2} \\
& +\left(\mathbf{m} \otimes \mathbf{n} \cdot \mathbf{C} \cdot \mathbf{n} \otimes \mathbf{m}-\rho c^{2}\right)=0 .
\end{aligned}
$$

The solution for Eq.(2.6) is:

$$
\gamma_{1,2}= \pm \sqrt{\frac{\rho c^{2}-\mathbf{m} \otimes \mathbf{n} \cdot \mathbf{C} \cdot \mathbf{n} \otimes \mathbf{m}}{\mathbf{m} \otimes \mathbf{v} \cdot \mathbf{C} \cdot \cdot \mathbf{v} \otimes \mathbf{m}}} .
$$

For the considered case, the general solution of Eq. (2.4) can be represented in the form:

$$
f\left(i r x^{\prime}\right)=C_{1} \sinh \left(i r \gamma x^{\prime}\right)+C_{2} \cosh \left(i r \gamma x^{\prime}\right),
$$

where $\gamma$ is the positive root in (2.7). For a Love wave in a substrate with attenuation condition (1.1) the considered solution becomes

$$
f\left(i r x^{\prime}\right)=C_{1} \exp \left(i r \gamma x^{\prime}\right)
$$

in representation (2.9) we chose the positive root for $\gamma$.

b) Supposing roots of the Christoffel equation (2.5) are multiple, we arrive at necessity to modify the solution of Eq. (2.4) by placing a logarithmic term (this corresponds to appearing one Jordan block in Eq. (2.4) that is reduced to a system of the first order). However, in the following analysis the case of multiple roots will not be studied; see [5,6] for the solutions related to multiple roots.

c) Representation (2.3) allows us to express surface tractions acting on any plane $x^{\prime}=$ const in the form:

$$
\mathbf{t}_{\mathbf{v}}(\mathbf{x})=\operatorname{ir} \mathbf{v} \cdot\left(\begin{array}{l}
\mathbf{C} \cdot \mathbf{v} f^{\prime}\left(i r x^{\prime}\right) \\
+\mathbf{C} \cdot \mathbf{n} f\left(i r x^{\prime}\right)
\end{array}\right) \cdot \mathbf{m} e^{i r(\mathbf{n} \cdot \mathbf{x}-c t)} .
$$

Assumption of monoclinic symmetry ensures surface tractions (2.10) to be collinear with vector $\mathbf{m}$. For an orthotropic material with axes of elastic symmetry coinciding with vectors $\mathbf{m}, \mathbf{n}$, and $\boldsymbol{v}$ expression (2.10) reduces to

$$
\mathbf{t}_{\mathbf{v}}(\mathbf{x})=i r \mathbf{v} \cdot \mathbf{C} \cdot \boldsymbol{v} \otimes \mathbf{m} f^{\prime}\left(i r x^{\prime}\right) e^{i r(\mathbf{n} \cdot \mathbf{x}-c t)} .
$$

As before, vector $\mathbf{t}_{\boldsymbol{v}}(\mathbf{x})$ is necessary collinear with vector $\mathbf{m}$. Expression (2.11) shows that on a boundary plane $x_{0}^{\prime}$ of a traction-free orthotropic plate the following condition must be satisfied: $f^{\prime}\left(i r x_{0}^{\prime}\right)=0$.

d) There is a major difference between bulk and surface $\mathrm{SH}$-waves. While both have the same polarization defined by vector $\mathbf{m}$, the corresponding stress components are different; see Figure 2. This concerns all kinds of surface $\mathrm{SH}$-waves including the genuine Love waves satisfying attenuation condition (1.1) and the so-called leakage Love waves propagating with speed $\left(c_{\mathbf{n m}}^{T}\right)_{\text {substrate }}$. The latter 
wave has the same speed and polarization as the corresponding bulk wave, does not attenuate in a substrate, but it is not a bulk wave in a substrate due to different stress components it generates. Indeed, for a surface SHwave propagating in an orthotropic medium the stress vector is defined by Eq. (2.11), while for a bulk SH-wave, the stress vector is:

$$
\mathbf{t}_{\mathbf{n}}=i r \mathbf{n} \cdot \mathbf{C} \cdot \mathbf{n} \otimes \mathbf{m} e^{i r(\mathbf{n} \cdot \mathbf{x}-c t)}
$$

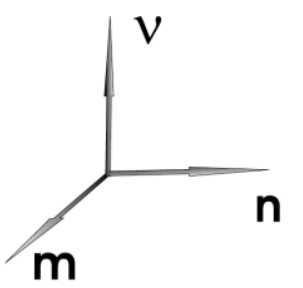

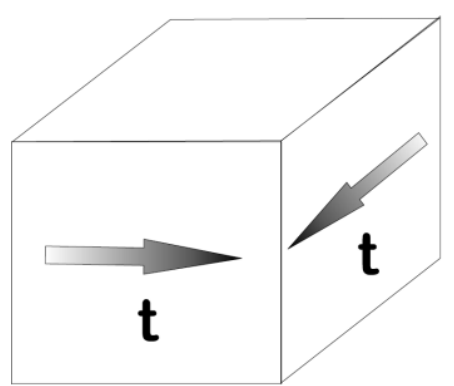

Bulk SH-wave

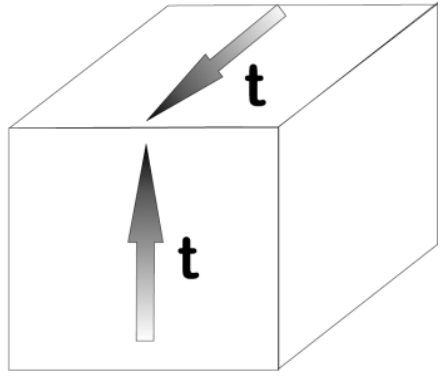

Surface SH-wave

Figure 2. Stresses for bulk and surface $\mathrm{SH}$-waves

\section{Energy of SH and Love Waves}

\subsection{Specific Kinetic and Elastic (potential) Energy}

Herein, we derive expressions for specific kinetic and elastic (potential) energy of the $\mathrm{SH}$-waves. Taking into account representation (2.3), the specific kinetic energy can be defined by:

$$
E_{k i n} \equiv \frac{1}{2} \rho \dot{\mathbf{u}} \cdot \overline{\dot{\mathbf{u}}}=\frac{1}{2} \rho \omega^{2}|f|^{2},
$$

where the following relation between the phase speed and frequency is used:

$$
\omega=r c .
$$

Equations (2.4), (3.1), and (3.2) allow us to represent the specific kinetic energy in the form:

$$
E_{k i n} \equiv \frac{1}{2} r^{2} \bar{f}\left[\begin{array}{l}
(\mathbf{m} \otimes \mathbf{v} \cdot \mathbf{C} \cdot \cdot \mathbf{v} \otimes \mathbf{m}) f^{\prime \prime} \\
+2(\mathbf{m} \cdot \operatorname{sym}(\mathbf{v} \cdot \mathbf{C} \cdot \mathbf{n}) \cdot \mathbf{m}) f^{\prime} \\
+(\mathbf{m} \otimes \mathbf{n} \cdot \mathbf{C} \cdot \mathbf{n} \otimes \mathbf{m}) f
\end{array}\right] .
$$

Another useful expression flows out from (3.1) and (3.2):

$$
\omega^{2}=\frac{2 E_{k i n}}{\rho|f|^{2}} .
$$

Similarly, the specific elastic energy can be defined by:

$$
\begin{aligned}
& E_{\text {elast }} \equiv \frac{1}{2} \nabla \mathbf{u} \cdot \mathbf{C} \cdot \cdot \nabla \overline{\mathbf{u}} \\
& =\frac{\omega^{2}}{2 c^{2}} \mathbf{m} \otimes\left(f^{\prime} \boldsymbol{v}+f \mathbf{n}\right) \cdot \mathbf{C} \cdot \cdot\left(\overline{f \mathbf{n}+f^{\prime} \boldsymbol{v}}\right) \otimes \mathbf{m} .
\end{aligned}
$$

REMARK 3.1. a) In view of Remark 2.2, expressions (3.1) and (3.5) coincide with the corresponding expressions for kinetic and elastic specific energy, obtained without using complex displacement fields.

b) Analysis of expressions (2.1), (2.3), (2.6), and (2.8) reveals that for the regarded waves $E_{\text {kin }} \neq E_{\text {elast }}$, due to presence of the generally non-constant function $f$. At the same time, for bulk waves $f=$ const, and hence from (2.1) and (2.3) we arrive at $E_{\text {kin }}=E_{\text {elast }}$; see also [2,3] for discussions.

PROPOSITION 3.1. a) If at some finite value of the phase speed the corresponding frequency $\omega$ vanishes, then both specific kinetic and elastic energies vanish also.

b) The specific kinetic energy vanishes at any $\omega$, on a plane $x^{\prime} \equiv \mathbf{v} \cdot \mathbf{x}=$ const, if function $f$ vanishes at this $x^{\prime}$.

c) The specific elastic energy does not vanish at any finite value of the phase speed and any non-vanishing frequency $\omega$.

Proofs of conditions a) and b) are obvious. Proof of condition c) follows from the positive definite condition for the elasticity tensor, Lemma 2.1, and expressions (3.2), (3.5).

\subsection{Group Speed}

The vector-valued group speed $\mathbf{v}_{\text {group }}$ is defined by $[2,3]$ :

$$
\mathbf{v}_{\text {group }}=\nabla_{(r \mathbf{n})} \omega
$$

where $\nabla_{(r \mathbf{n})}$ denotes gradient with respect to the independent spatial variable $(r \mathbf{n})$. For the subsequent analysis the scalar group speed $c_{\text {group }}$ will also be needed: 


$$
c_{\text {group }} \equiv\left|\mathbf{v}_{\text {group }}\right|=\sqrt{\nabla_{(r \mathbf{n})} \omega \cdot \overline{\nabla_{(r \mathbf{n})} \omega}} .
$$

Now, combining (3.3), (3.4), and (3.7) yields:

$$
c_{\text {group }}=\frac{\sqrt{\left(f^{\prime} \mathbf{v}+f \mathbf{n}\right) \cdot(\mathbf{m} \cdot \mathbf{C} \cdot \mathbf{m})^{2} \cdot\left(\overline{f \mathbf{n}+f^{\prime} \mathbf{v}}\right)}}{c \rho|f|},
$$

where as before, $c$ stands for the phase speed.

PROPOSITION 3.2. a) At any physically admissible properties of a medium and any $\mathrm{SH}$-wave propagating with the finite phase speed $c \neq 0$, the corresponding group speed $c_{\text {group }}$ is delimited from zero.

b) If $f \rightarrow 0$ at $x^{\prime} \rightarrow x_{0}^{\prime}$, where $x_{0}^{\prime}$ takes some finite value, then $c_{\text {group }} \rightarrow \infty$.

Proof a) flows out from observation that the radicand in (3.8) is strictly positive due to (2.2) and Lemma 2.1. Proof b) is obvious.

\subsection{Ray Speed}

The vector-valued ray speed can be defined by (see [3]):

$$
\mathbf{v}_{\text {ray }}=\frac{\mathbf{J}_{\text {elast }}}{E_{\text {kin }}+E_{\text {elast }}},
$$

where $\mathbf{J}_{\text {elast }}$ is the flux of elastic energy:

$$
\mathbf{J}_{\text {elast }} \equiv \dot{\mathbf{u}} \cdot \mathbf{C} \cdot \nabla \mathbf{u}
$$

The corresponding scalar ray speed is:

$$
c_{\text {ray }} \equiv\left|\mathbf{v}_{\text {ray }}\right|=\frac{\sqrt{\mathbf{J}_{\text {elast }} \cdot \overline{\mathbf{J}_{\text {elast }}}}}{E_{\text {kin }}+E_{\text {elast }}}
$$

Substituting (2.3) into (3.11) and exploiting (3.3), (3.5), yields:

$$
c_{\text {ray }}=\frac{2 c|f| \sqrt{\left(f^{\prime} \mathbf{v}+f \mathbf{n}\right) \cdot(\mathbf{m} \cdot \mathbf{C} \cdot \mathbf{m})^{2} \cdot\left(\overline{f \mathbf{n}+f^{\prime} \mathbf{v}}\right)}}{\rho c^{2}|f|^{2}+\left(f^{\prime} \mathbf{v}+f \mathbf{n}\right) \cdot(\mathbf{m} \cdot \mathbf{C} \cdot \mathbf{m}) \cdot\left(\overline{f \mathbf{n}+f^{\prime} \mathbf{v}}\right)}
$$

PROPOSITION 3.3. a) At any physically admissible properties of a medium and any $\mathrm{SH}$-wave propagating with the finite phase speed $c \neq 0$, the corresponding ray speed $c_{\text {ray }}$ is delimited from zero.

b) If $f \rightarrow 0$ at $x^{\prime} \rightarrow x_{0}^{\prime}$, where $x_{0}^{\prime}$ is finite, then $c_{\text {ray }} \rightarrow \infty$.

c) A necessary and sufficient condition for $c_{\text {group }}=c_{\text {ray }}$, is as follows:

$$
E_{\text {kin }}=E_{\text {elast }} \text {. }
$$

Proofs a) and b) are analogous to the proof of Proposition 3.2. Proof c) follows directly from (3.8), (3.12), with account of (3.1), (3.5).

\section{SH-waves in a Single-layered Orthotropic Plate}

Hence it will be assumed that vectors $\mathbf{v}, \mathbf{m}$, and $\mathbf{n}$ coincide with the axes of elastic symmetry of an orthotropic medium.
REMARK 4.1. It can be shown (see [6]) that regardless of boundary conditions and at imaginary roots of Eq. (2.6), no $\mathrm{SH}$-wave can propagate in directions of elastic symmetry of an orthotropic single-layered plate. Thus, the following inequality

$$
c>\sqrt{\frac{\mathbf{m} \otimes \mathbf{n} \cdot \mathbf{C} \cdot \mathbf{n} \otimes \mathbf{m}}{\rho}},
$$

naturally arising from (2.7), delivers a necessary condition for existing surface $\mathrm{SH}$-wave. Thus, for the regarded plate all surface $\mathrm{SH}$-waves are necessary supersonic, since the radicand in the right-hand side of (4.1) defines speed of the corresponding shear bulk wave $c_{\mathbf{n m}}^{T}$. In this section we assume condition (4.1) to hold.

\subsection{Traction-free Plate}

Herein we consider a single-layered plate with the traction-free boundary conditions:

$$
\left\{\begin{array}{l}
\mathbf{t}_{\boldsymbol{v}}(h / 2)=0 \\
\mathbf{t}_{\boldsymbol{v}}(-h / 2)=0
\end{array},\right.
$$

where $h$ is the thickness of the plate (we choose origin of coordinates at the median plane).

For such a plate, finding function $f$ from (2.6), (4.2), yields:

$$
f\left(i r x^{\prime}\right)=\left\{\begin{array}{l}
\cos \left(r \gamma x^{\prime}\right), \text { at } r=\frac{2 n \pi}{\gamma h} \\
\sin \left(r \gamma x^{\prime}\right), \text { at } r=\frac{(2 n-1) \pi}{\gamma h}
\end{array} \quad n=1,2, \ldots,\right.
$$

where $\gamma$ is defined by (2.7).

PROPOSITION 4.1. a) On planes $x^{\prime}=$ const , where

$x^{\prime}=\left\{\begin{array}{ll}\frac{\frac{1}{2}+k}{2 n} h, \quad \text { at } r=\frac{2 n \pi}{\gamma h}, \quad-n \leq k<n \\ \frac{k}{2 n-1} h, \quad \text { at } r=\frac{(2 n-1) \pi}{\gamma h},-n \leq k<n\end{array} \quad n, k \in \mathbb{Z},(4.4)\right.$

both the displacement field and specific kinetic energy vanish. That is equivalent to existence of the internal immovable layers under propagating $\mathrm{SH}$-wave on a traction-free plate.

b) At any finite phase speed satisfying inequality (4.1), there are no waves propagating at vanishing frequency (both phase speed and frequency are delimited from zero).

Proof a) follows from considering zeroes of the function, defined by (4.3). Proof b) follows from analyzing expressions (4.3), (3.2). It reveals that no nontrivial solutions exist at $\omega=0$.

\subsection{Clamped Plate}

For a single-layered plate with clamped outer surfaces, boundary conditions are:

$$
\left\{\begin{array}{l}
\mathbf{u}(h / 2)=0 \\
\mathbf{u}(-h / 2)=0
\end{array} .\right.
$$

Finding function $f$ from Eq. (2.4) and satisfying boundary conditions (4.5), yields: 


$$
f\left(i r x^{\prime}\right)=\left\{\begin{array}{l}
\sin \left(r \gamma x^{\prime}\right), \text { at } r=\frac{2 n \pi}{\gamma h} \\
\cos \left(r \gamma x^{\prime}\right), \text { at } r=\frac{(2 n-1) \pi}{\gamma h}
\end{array} \quad n=1,2, \ldots\right. \text { (4.6) }
$$

Similarly to Proposition 4.1, we have

PROPOSITION 4.2. a) On planes $x^{\prime}=$ const , where

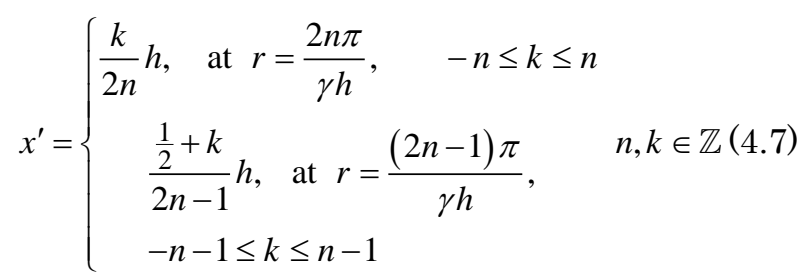

both the displacement field and specific kinetic energy vanish. That is equivalent to existence of the internal immovable layers under propagating surface $\mathrm{SH}$-wave on a clamped plate.

b) At any finite phase speed satisfying inequality (4.1), there are no waves propagating at vanishing frequency (both phase speed and frequency are delimited from zero).

\subsection{Plate with Mixed Boundary Conditions}

Herein we consider a plate with traction-free upper and clamped bottom surface:

$$
\left\{\begin{array}{l}
\mathbf{t}_{\mathbf{v}}(h / 2)=0 \\
\mathbf{u}(-h / 2)=0
\end{array} .\right.
$$

Direct analysis reveals that function $f$ satisfying homogeneous boundary conditions (4.8) takes the form:

$$
f\left(i r x^{\prime}\right)=\left\{\begin{array}{l}
\sin \left(r \gamma x^{\prime}-\frac{\pi}{4}\right), \text { at } r=\frac{2\left(n-\frac{1}{4}\right) \pi}{\gamma h}, n=1,2, \ldots \\
\sin \left(r \gamma x^{\prime}+\frac{\pi}{4}\right), \text { at } r=\frac{2\left(n+\frac{1}{4}\right) \pi}{\gamma h}, n=0,1, \ldots
\end{array}\right.
$$

Proposition 4.3. a) On planes $x^{\prime}=$ const , where

$$
x^{\prime}=\left\{\begin{array}{l}
\frac{\left(k+\frac{1}{4}\right)}{2\left(n-\frac{1}{4}\right)} h, \text { at } r=\frac{2\left(n-\frac{1}{4}\right) \pi}{\gamma h},-n \leq k<n \\
\frac{\left(k-\frac{1}{4}\right)}{2\left(n+\frac{1}{4}\right)} h, \text { at } r=\frac{2\left(n+\frac{1}{4}\right) \pi}{\gamma h},-n \leq k \leq n
\end{array} \quad n, k \in \mathbb{Z}(4.10)\right.
$$

both the displacement field and specific kinetic energy vanish. That is equivalent to existence of immovable layers under propagating surface $\mathrm{SH}$-wave on a clamped plate.

b) At any finite phase speed satisfying inequality (4.1), there are no waves propagating at vanishing frequency (both phase speed and frequency are delimited from zero).

\subsection{Concluding Remarks}

For monoclinic single-layered plates and all the considered boundary conditions (i) the admissible speed interval is transonic:

$$
c \in\left(c_{\mathbf{m n}}^{T} ; \infty\right)
$$

(ii) at any phase speed satisfying (4.11) there are immovable longitudinal layers, and (iii) there are no anomalous $\mathrm{SH}$-waves corresponding to vanishing frequency.

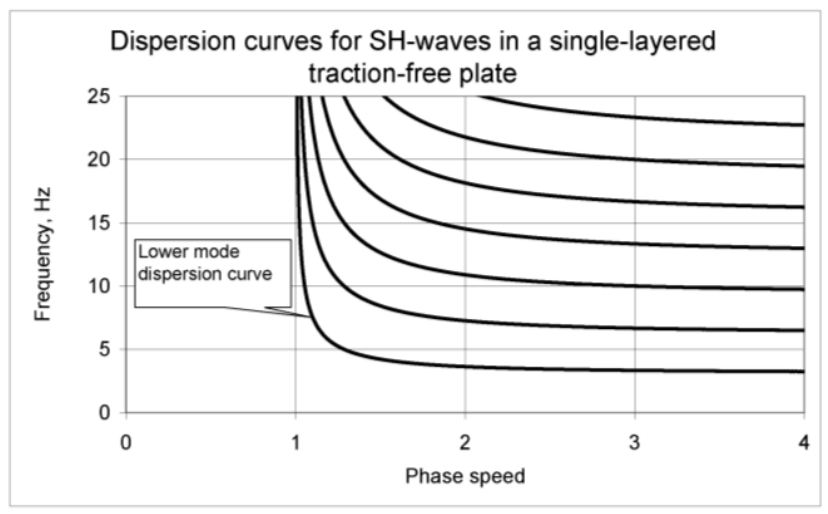

Figure 3. Dispersion curves for $\mathrm{SH}$-waves propagating in a singlelayered traction-free orthotropic plate with $\left(c_{\mathbf{n m}}^{T}\right)=1$

Below, the dispersion relations will be plotted in terms of the phase speed $c$ and frequency $\omega$, or the phase speed and wave number $r$, the latter is connected with $c$ and $\omega$ by (3.2). The typical dispersion curves for a homogeneous traction-free plate are presented in Figure 3.

These curves relate to a plate with $h=1, c_{\mathbf{n m}}^{T}=1$. The lower mode dispersion curves for this plate but with different boundary conditions are plotted in Figure 4.

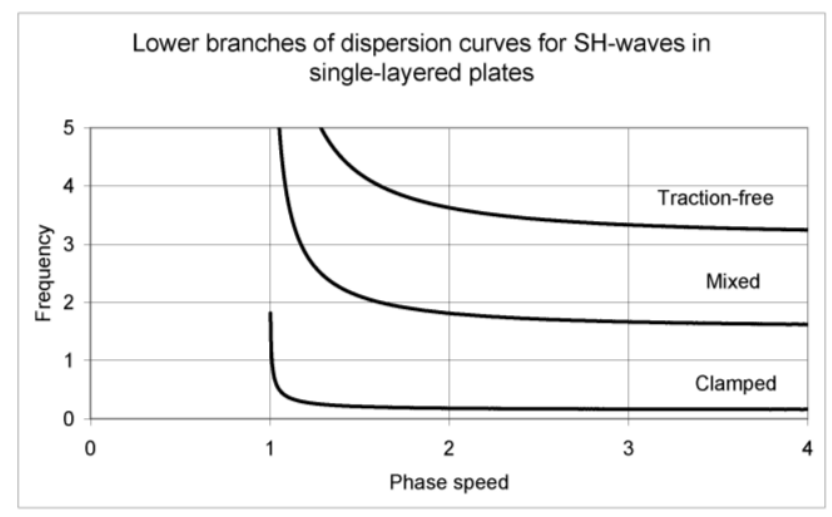

Figure 4. Lower branches of dispersion curves for SH-waves propagating in a single layered orthotropic plate $\left(c_{\mathbf{n m}}^{T}\right)=1$ with different boundary conditions

It should also be noted that a lower mode wave is usually the easiest to launch, and it appears to be the most informative due to its dispersion. The presented data reveal also that for a given plate its boundary conditions can be distinguished easily by analyzing lower mode dispersion curves.

\section{Love Waves}

In this section we assume inequality (1.2) to hold ensuring existence of Love wave. Different reference coordinate systems for the layer (with the origin at the median plane) and for the substrate (with the origin at the interface) will be chosen. 


\subsection{Analytical Solutions}

For Love wave we consider the following boundary conditions: (i) the traction-free boundary conditions at the outer surface of a layer, (ii) contact type conditions at the interface, and (iii) the attenuation condition (1.1) at $x^{\prime} \rightarrow-\infty$ :

$$
\left\{\begin{array}{l}
\left.\mathbf{t}_{\mathbf{v}}\left(h_{1} / 2\right)\right|_{1}=0 \\
\left.\mathbf{t}_{\mathbf{v}}\left(-h_{1} / 2\right)\right|_{1}=-\left.\mathbf{t}_{-\mathbf{v}}(0)\right|_{2} ;\left.\quad \mathbf{u}\left(-h_{1} / 2\right)\right|_{1}=\left.\mathbf{u}(0)\right|_{2} \\
\left.\mathbf{u}\left(x^{\prime}\right)\right|_{2} \rightarrow 0 \text { at } \quad x^{\prime} \rightarrow-\infty
\end{array}\right.
$$

In Eqs. (5.1) and further index 1 is referred to the layer, and 2 to the substrate.

Applying the Modified Transfer Matrix (MTM) method [5], functions $f_{k}, k=1,2$, which define the corresponding displacement fields, can be represented in the form:

$$
f_{k}\left(i r x^{\prime}\right)= \begin{cases}\sin \left(r \gamma_{1}\left(x^{\prime}-h_{1} / 2\right)\right), & k=1 \\ \exp \left(i r \gamma_{2} x^{\prime}\right), & k=2\end{cases}
$$

The implicit secular equation for the wave number can be written in the form [5]:

$$
\left(\begin{array}{l}
\left(\mathbf{m} \otimes \mathbf{v} \cdot \mathbf{C}_{1} \cdot \cdot \boldsymbol{v} \otimes \mathbf{m}\right) \gamma_{1} \sinh \left(i r \gamma_{1} h_{1}\right) \\
-\left(\mathbf{m} \otimes \mathbf{v} \cdot \mathbf{C}_{2} \cdot \mathbf{v} \otimes \mathbf{m}\right) \gamma_{2} \cosh \left(i r \gamma_{1} h_{1}\right)
\end{array}\right)=0
$$

Proposition 5.1. a) Suppose that

$$
\left(c_{\mathbf{n m}}^{T}\right)_{1}<c<\left(c_{\mathbf{n m}}^{T}\right)_{2},
$$

then on planes $x^{\prime}=$ const (in a layer), where

$$
\begin{aligned}
& x^{\prime}=\frac{n \pi}{r \gamma_{1}}+\frac{h_{1}}{2}, \quad n \in \mathbb{Z}, \\
& -E n t\left(\frac{r \gamma_{1} h_{1}}{\pi}\right) \leq n \leq 0
\end{aligned}
$$

and $r$ satisfies Eq. (5.3), both the displacement field and specific kinetic energy vanish.

b) No Love waves propagate, when

$$
c<\left(c_{\mathbf{n m}}^{T}\right)_{1} \text { or } c>\left(c_{\mathbf{n m}}^{T}\right)_{2}
$$

c) At the phase speed $c \rightarrow\left(c_{\mathbf{n m}}^{T}\right)_{2}-0$, there is a lower mode leakage Love wave propagating with vanishing wave number.

Proof a) flows out from analyzing expression (5.2) for function $f_{1}$. Values for $x^{\prime}$ defined by (5.5) and (5.6), are zeroes of this function.

Proof b) follows from an observation that at the phase speed satisfying the first inequality in (5.7), both $\gamma_{1}$ and $\gamma_{2}$ are imaginary due to (2.7) and (1.2). But, Eq. (5.3) at imaginary $\gamma_{k}, k=1,2$ reduces to

$$
\tanh \left(r\left|\gamma_{1}\right| h_{1}\right)=-\frac{\left(\mathbf{m} \otimes \mathbf{v} \cdot \mathbf{C}_{2} \cdot \mathbf{v} \otimes \mathbf{m}\right)\left|\gamma_{2}\right|}{\left(\mathbf{m} \otimes \mathbf{v} \cdot \mathbf{C}_{1} \cdot \mathbf{v} \otimes \mathbf{m}\right)\left|\gamma_{1}\right|} .
$$

The latter equation has no positive solutions with respect to the wave number $r$. Now, at the phase speed satisfying the second inequality in (5.7), we see that the exponent term in $(5.2)_{2}$ does not satisfy the attenuating condition at $x^{\prime} \rightarrow \infty$.
To prove c) we need to consider Eq. (5.3) at small $r$ :

$$
\begin{aligned}
& \left(\mathbf{m} \otimes \mathbf{v} \cdot \mathbf{C}_{2} \cdot \mathbf{v} \otimes \mathbf{m}\right) \gamma_{2} \\
& +\left(-i\left(\mathbf{m} \otimes \mathbf{v} \cdot \mathbf{C}_{1} \cdot \mathbf{v} \otimes \mathbf{m}\right) \gamma_{1}{ }^{2} h_{1}\right) r+O\left(r^{2}\right)=0 .
\end{aligned}
$$

Supposing that $c \rightarrow\left(c_{\mathbf{n m}}^{T}\right)_{2}-0$, which implys $\operatorname{Im}\left(\gamma_{2}\right) \rightarrow 0, \operatorname{Re}\left(\gamma_{2}\right)=0$, and letting $r \rightarrow 0$, which gives a wave corresponding to the lower branch, we arrive at the following condition:

$$
f_{2} \rightarrow \text { const }
$$

uniformly on any compact in $(-\infty, 0]$. The latter proves condition c).

REMARK 5.1. a) However, leakage Love wave introduced in Proposition 5.1.c, does not coincide with the horizonally polarized bulk wave in the substrate. That is because leakage Love wave travelling with the same speed $\left(c_{\mathbf{n m}}^{T}\right)_{2}$ as the shear bulk wave in the substrate (or better speaking, travelling with the phase speed $\left.c \rightarrow\left(c_{\mathbf{n m}}^{T}\right)_{2}-0\right)$, leads to different stress components; see Figure 2. Moreover, as expresion (2.11) shows this leakage wave is a stress-free wave.

b) Another interesting observation concerns amplitude distribiution along depth of a layer and substrate. According to representations (2.3) and (5.2), the distribution in both layer and substrate tends to a constant $\mathbf{m}$ as $c \rightarrow\left(c_{\mathbf{n m}}^{T}\right)_{2}-0$.

\subsection{Numerical Example}

Herein we consider an orthotropic layer lying on an orthotropic substrate. The outer plane (Figure 1) of the layer is traction-free. The layer and substrate are characterized by the following parameters:

$$
\left(c_{\mathbf{n m}}^{T}\right)_{1}=1, \quad h_{1}=1, \quad\left(c_{\mathbf{n m}}^{T}\right)_{2}=2 .
$$

The corresponding dispersion curves are presented in Figure 5 .

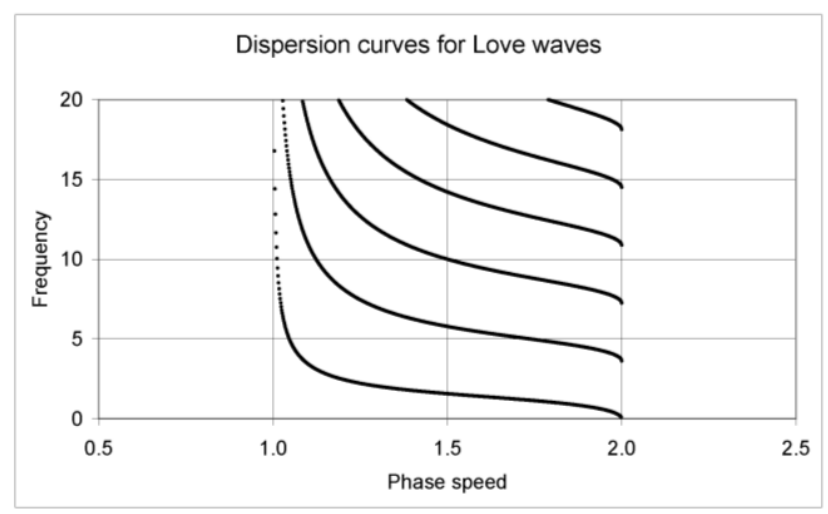

Figure 5. Dispersion curves for Love waves propagating in an orthotropic medium with $\left(c_{\mathbf{n m}}^{T}\right)_{\text {layer }}=1 ;\left(c_{\mathbf{n m}}^{T}\right)_{\text {substrate }}=2$

These data demonstrate presence of a lower mode leakage wave [5] propagating with the phase speed $c \rightarrow\left(c_{\mathbf{n m}}^{T}\right)_{2}-0$ and having vanishing frequency. 


\section{Two-layered Orthotropic Plates}

It is assumed that (i) both layers are orthotropic with axes of elastic symmetry coincident with vectors $\mathbf{n}, \mathbf{v}$, and $\mathbf{m}$; and (ii) the corresponding shear bulk waves differ:

$$
\left(c_{\mathbf{n m}}^{T}\right)_{1} \neq\left(c_{\mathbf{n m}}^{T}\right)_{2} .
$$

REMARK 6.1. a) If inequality (6.1) violates, then the two-layered plate appears as a single-layered, with respect to the considered $\mathrm{SH}$-wave.

b) In this section and further we assume that each layer has its own reference coordinate system with the origin lying in the median plane of a layer.

\subsection{Traction-free Plate}

Boundary conditions for a traction-free plate are:

$$
\left\{\begin{array}{l}
t_{v}\left(h_{1} / 2\right)=0 \\
t_{v}\left(-h_{2} / 2\right)=0
\end{array},\right.
$$

where lower indicies are referred to the corresponding layers.

Applying the MTM method [5,6], functions $f_{k}, k=1,2$ which define the displacement field in the corresponding layeres, can be represented in the form:

$$
f_{k}\left(i r x^{\prime}\right)=\cos \left(r \gamma_{k}\left(x^{\prime}+(-1)^{k} h_{k} / 2\right)\right), \quad k=1,2
$$

at the wave number $r$ satisfying the following equation [6]:

$$
\begin{aligned}
& \frac{\left(\mathbf{m} \otimes \boldsymbol{v} \cdot \mathbf{C}_{1} \cdot \boldsymbol{v} \otimes \mathbf{m}\right) \gamma_{1}}{\left(\mathbf{m} \otimes \boldsymbol{v} \cdot \mathbf{C}_{2} \cdot \boldsymbol{v} \otimes \mathbf{m}\right) \gamma_{2}} \sin \left(r \gamma_{1} h_{1}\right) \cos \left(r \gamma_{2} h_{2}\right) \\
& +\cos \left(r \gamma_{1} h_{1}\right) \sin \left(r \gamma_{2} h_{2}\right)=0 .
\end{aligned}
$$

Proposition 6.1. a) Suppose that

$$
\min \left(\left(c_{\mathbf{n m}}^{T}\right)_{1} ;\left(c_{\mathbf{n m}}^{T}\right)_{2}\right)<c<\max \left(\left(c_{\mathbf{n m}}^{T}\right)_{1} ;\left(c_{\mathbf{n m}}^{T}\right)_{2}\right)
$$

where $\left(c_{\mathbf{n m}}^{T}\right)_{k}$ is the bulk wave speed in the corresponding layer, then on planes $x^{\prime}=$ const where

$$
x^{\prime}=\frac{\pi(1+2 n)}{2 r \gamma_{k}}-(-1)^{k} \frac{h_{k}}{2}, \quad n \in \mathbb{Z},
$$

and

$$
\begin{aligned}
& -\operatorname{Ent}\left(\left(1-(-1)^{k}\right) \frac{r \gamma_{k} h_{k}}{2 \pi}+\frac{1}{2}\right) \\
& \leq n \leq \operatorname{Ent}\left(\left(1+(-1)^{k}\right) \frac{r \gamma_{k} h_{k}}{2 \pi}-\frac{1}{2}\right)
\end{aligned}
$$

and $r$ satisfies Eq. (6.4)), both the displacement field and specific kinetic energy vanish in a layer with the minimal bulk wave speed $c_{\mathbf{n m}}^{T}$.

b) Suppose that

$$
c>\max \left(\left(c_{\mathbf{n m}}^{T}\right)_{1} ;\left(c_{\mathbf{n m}}^{T}\right)_{2}\right),
$$

(the phase speed is transonic in both layers), then on planes $x^{\prime}=$ const where $x^{\prime}$ satisfies Eq. (6.6) and $n$ satisfies Eq. (6.7), the displacement field and specific kinetic energy vanish in both layers.

c) At the phase speed $c \rightarrow c_{s}-0$, where

$c_{s}=\sqrt{\frac{\left(\mathbf{m} \otimes \mathbf{n} \cdot \mathbf{C}_{1} \cdot \mathbf{n} \otimes \mathbf{m}\right) h_{1}+\left(\mathbf{m} \otimes \mathbf{n} \cdot \mathbf{C}_{2} \cdot \mathbf{n} \otimes \mathbf{m}\right) h_{2}}{\rho_{1} h_{1}+\rho_{2} h_{2}}}$,

there is a lower mode $\mathrm{SH}$-wave propagating with vanishing wave number $r \rightarrow 0$.

Proofs a) and b) flow out from expression (6.3) for functions $f_{k}$. Values for $x^{\prime}$ defined by (6.6) and (6.7), are zeroes of these functions.

To prove c) we need to consider Eq. (6.4) at small $r$ :

$$
\left(\begin{array}{l}
\left(\mathbf{m} \otimes \mathbf{v} \cdot \mathbf{C}_{1} \cdot \boldsymbol{v} \otimes \mathbf{m}\right) \gamma_{1}^{2} h_{1} \\
+\left(\mathbf{m} \otimes \mathbf{v} \cdot \mathbf{C}_{2} \cdot \mathbf{v} \otimes \mathbf{m}\right) \gamma_{2}{ }^{2} h_{2}
\end{array}\right) r+O\left(r^{3}\right)=0 .
$$

Equating to zero the coefficient at $r$ in the left-hand side of Eq. (6.10), we arrive at the solution for the phase speed given by (6.9).

DEFINITION 6.1. A lower mode surface SH-wave propagating with the phase speed $c_{s}$ and with vanishing wave number will be called the anomalous surface wave.

Proposition 6.1 along with expressions (3.1), (3.2), (3.5) ensure:

COROLlARY 6.1. a) Both kinetic and potential energy vanish at the anomalous surface wave.

b) At sufficiently small $r$ and real $\gamma_{k}$ in both planes there can be no planes with vanishing displacement field (all $\mathrm{SH}$-waves in the vicinity of the anomalous $\mathrm{SH}$-wave do not have planes at which the displacement field vanishes).

REMARK 6.2. a) Proposition 6.1 shows that planes with vanishing kinetic energy arise only if the phase speed becomes transonic for the corresponding layer.

b) Direct analysis reveals that the wave speed $c_{s}$ satisfies the inequalities:

$\min \left(\left(c_{\mathbf{n m}}^{T}\right)_{1} ;\left(c_{\mathbf{n m}}^{T}\right)_{2}\right) \leq c_{s} \leq \max \left(\left(c_{\mathbf{n m}}^{T}\right)_{1} ;\left(c_{\mathbf{n m}}^{T}\right)_{2}\right)$

c) The considered $\mathrm{SH}$-waves in the vicinty of the anomalous $\mathrm{SH}$-wave resemble solitones, since their wave number tends to zero, as $c \rightarrow c_{s}-0$.

d) Taking into account Eqs. (3.1), (3.5), we have for the lower branch of the $\mathrm{SH}$-waves at $c \rightarrow c_{S}-0$ :

$$
E_{\text {kin }} \rightarrow 0, \quad E_{\text {elast }} \rightarrow 0 .
$$

In obtaining (6.12), restriction of $\|f\|_{2}^{1}$ at $c \rightarrow c_{s}-0$ is accounted (herein $\|\cdot\|_{2}^{1}$ denotes the corresponding Hörmander's norm). Thus, conditions (6.12) ensure minimum energy required to initiate lower branch of the $\mathrm{SH}$-wave at $c \rightarrow c_{s}-0$. Moreover, similarly to the leakage Love wave discussed in Remark 5.1, the lower branch of the $\mathrm{SH}$-waves at $c \rightarrow c_{s}-0$ is a stress-free wave, and the amplitude distribution in both layers tends to a constant $\mathbf{m}$ at $c \rightarrow c_{s}-0$.

\subsection{Clamped Plate}


Boundary conditions for a clamped plate are:

$$
\left\{\begin{array}{l}
\mathbf{u}\left(h_{1} / 2\right)=0 \\
\mathbf{u}\left(-h_{2} / 2\right)=0
\end{array}\right.
$$

Application of the Modified Transfer Matrix (MTM) method gives functions $f_{k}, k=1,2$ in the form:

$$
f_{k}\left(i r x^{\prime}\right)=\sin \left(r \gamma_{k}\left(x^{\prime}+(-1)^{k} h_{k} / 2\right)\right), \quad k=1,2(6.14)
$$

The wave number in representation (6.14) satisfies the following equation [6]:

$$
\begin{aligned}
& \frac{\left(\mathbf{m} \otimes \boldsymbol{v} \cdot \mathbf{C}_{2} \cdot \boldsymbol{v} \otimes \mathbf{m}\right) \gamma_{2}}{\left(\mathbf{m} \otimes \boldsymbol{v} \cdot \mathbf{C}_{1} \cdot \boldsymbol{v} \otimes \mathbf{m}\right) \gamma_{1}} \sin \left(r \gamma_{1} h_{1}\right) \cos \left(r \gamma_{2} h_{2}\right) \\
& +\cos \left(r \gamma_{1} h_{1}\right) \sin \left(r \gamma_{2} h_{2}\right)=0
\end{aligned}
$$

Similarly to the preceding case, we have PROPOSITION 6.2. a) Suppose that

$$
\min \left(\left(c_{\mathbf{n m}}^{T}\right)_{1} ;\left(c_{\mathbf{n m}}^{T}\right)_{2}\right)<c<\max \left(\left(c_{\mathbf{n m}}^{T}\right)_{1} ;\left(c_{\mathbf{n m}}^{T}\right)_{2}\right),
$$

then on planes $x^{\prime}=$ const where

$$
x^{\prime}=\frac{n \pi}{r \gamma_{k}}-(-1)^{k} \frac{h_{k}}{2}, \quad n \in \mathbb{Z},
$$

and

$$
\left\{\begin{array}{c}
-E n t\left(\frac{r \gamma_{1} h_{1}}{\pi}\right) \leq n \leq 0, \quad \text { if }\left(c_{\mathbf{n m}}^{T}\right)_{1}<\left(c_{\mathbf{n m}}^{T}\right)_{2} \\
\text { or } \\
0 \leq n \leq E n t\left(\frac{r \gamma_{2} h_{2}}{\pi}\right), \quad \text { if }\left(c_{\mathbf{n m}}^{T}\right)_{1}>\left(c_{\mathbf{n m}}^{T}\right)_{2}
\end{array}\right.
$$

and $r$ satisfies Eq. (6.4)), both the displacement field and specific kinetic energy vanish in a layer with the minimal bulk wave speed $c_{\mathbf{n m}}^{T}$.

b) Suppose that

$$
c>\max \left(\left(c_{\mathbf{n m}}^{T}\right)_{1} ;\left(c_{\mathbf{n m}}^{T}\right)_{2}\right),
$$

(the phase speed is transonic for both layers), then on planes $x^{\prime}=$ const where

$$
\begin{aligned}
& x^{\prime}=\frac{n \pi}{r \gamma_{k}}-(-1)^{k} \frac{h_{k}}{2}, \quad n \in \mathbb{Z} \\
& \text { and }\left\{\begin{array}{cl}
-E n t\left(\frac{r \gamma_{1} h_{1}}{\pi}\right) \leq n \leq 0, & \text { at } k=1 \\
\text { and } & \text { at } k=2
\end{array}\right. \text {, }
\end{aligned}
$$

the displacement field and specific kinetic energy vanish.

c) At the phase speed $c \rightarrow c_{s}-0$, where $c_{s}$ satisfies Eq. (6.9), there is a lower mode $\mathrm{SH}$-wave propagating with vanishing wave number $r \rightarrow 0$.

\subsection{Plate with Mixed Boundary Conditions}

Boundary conditions for the considered plate are:

$$
\left\{\begin{array}{l}
\mathbf{t}_{\mathbf{v}}\left(h_{1} / 2\right)=0 \\
\mathbf{u}\left(-h_{2} / 2\right)=0
\end{array} .\right.
$$

Application of the Modified Transfer Matrix (MTM) method gives functions $f_{k}, k=1,2$ in the form:

$$
f_{k}\left(i r x^{\prime}\right)=\left\{\begin{array}{ll}
\cos \left(r \gamma_{1}\left(x^{\prime}-h_{1} / 2\right)\right), & k=1 \\
\sin \left(r \gamma_{2}\left(x^{\prime}+h_{2} / 2\right)\right), & k=2
\end{array} .\right.
$$

For the considered case, the wave number satisfies the following equation [6]:

$$
\begin{aligned}
& \frac{\left(\mathbf{m} \otimes \boldsymbol{v} \cdot \mathbf{C}_{2} \cdot \boldsymbol{v} \otimes \mathbf{m}\right) \gamma_{2}}{\left(\mathbf{m} \otimes \boldsymbol{v} \cdot \mathbf{C}_{1} \cdot \cdot \boldsymbol{v} \otimes \mathbf{m}\right) \gamma_{1}} \cos \left(r \gamma_{1} h_{1}\right) \cos \left(r \gamma_{2} h_{2}\right) \\
& -\sin \left(r \gamma_{1} h_{1}\right) \sin \left(r \gamma_{2} h_{2}\right)=0
\end{aligned}
$$

Similarly to the preceding cases, we have

Proposition 6.3. a) Suppose that

$\min \left(\left(c_{\mathbf{n m}}^{T}\right)_{1} ;\left(c_{\mathbf{n m}}^{T}\right)_{2}\right)<c<\max \left(\left(c_{\mathbf{n m}}^{T}\right)_{1} ;\left(c_{\mathbf{n m}}^{T}\right)_{2}\right)$,

then on planes $x^{\prime}=$ const where $x^{\prime}$ satisfies Eqs. (6.17), (6.18), both the displacement field and specific kinetic energy vanish in a layer with the minimal bulk wave speed $c_{\mathbf{n m}}^{T}$.

b) Suppose that

$$
c>\max \left(\left(c_{\mathbf{n m}}^{T}\right)_{1} ;\left(c_{\mathbf{n m}}^{T}\right)_{2}\right),
$$

(the phase speed is transonic for both layers), then on planes $x^{\prime}=$ const where $x^{\prime}$ satisfies Eqs (6.20), the displacement field and specific kinetic energy vanish in both layers.

c) At the phase speed $c \rightarrow c_{s}-0$, where $c_{s}$ satisfies Eq. (6.9), there is a lower mode $\mathrm{SH}$-wave propagating with vanishing wave number $r \rightarrow 0$.

\subsection{Numerical Examples}

Thus, for a two-layered monoclinic plate with all the considered boundary conditions (i) the admissible speed interval is partly transonic

$$
c \in\left(\min \left(\left(c_{\mathbf{m n}}^{T}\right)_{1},\left(c_{\mathbf{m n}}^{T}\right)_{2}\right) ; \infty\right)
$$

(ii) at any phase speed satisfying (6.26) there are immovable longitudinal planes, and (iii) there are anomalous lower-mode $\mathrm{SH}$-waves at $c \rightarrow c_{s}-0$.

Typical dispersion curves (in terms of the frequency $\omega$ and phase speed $c$ ) for a two-layered traction-free plate with

$$
h_{1}=h_{2}=1,\left(c_{\mathbf{n m}}^{T}\right)_{1}=1,\left(c_{\mathbf{n m}}^{T}\right)_{2}=2,
$$

are presented in Figure 6.

Existence of the anomalous $\mathrm{SH}$-wave at $c \rightarrow c_{s}-0$ $\left(c_{s} \approx 1.59\right)$ propagating in a two-layered plate, resembles a lower mode leakage Love wave propagating with vanishing frequency at the speed

$$
c \rightarrow\left(c_{\mathbf{m n}}^{T}\right)_{\text {substrate }}-0 ;
$$


see Remark 5.1 and Figure 5.

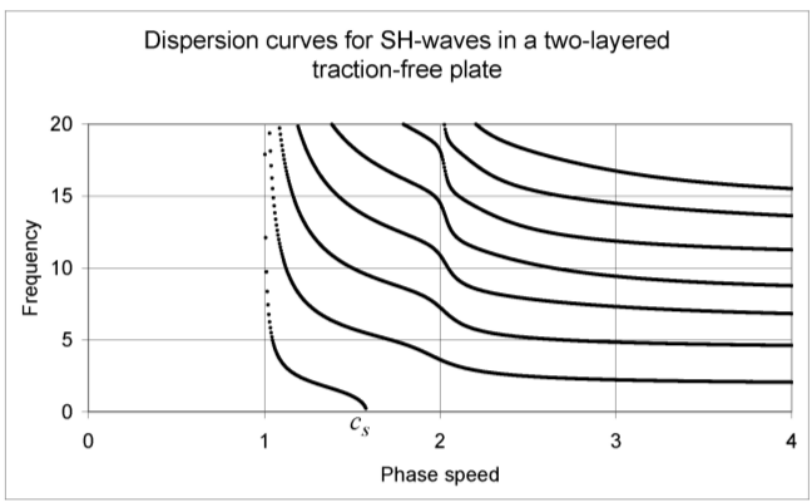

Figure 6. Dispersion curves for SH-waves propagating in a two-layered traction-free orthotropic plate with $\left(c_{\mathbf{n m}}^{T}\right)_{1}=1 ;\left(c_{\mathbf{n m}}^{T}\right)_{2}=2$, and $h_{1}=h_{2}=1$

We also analyzed influence of the depth increase of a bottom layer in a two-layered plate on behavior of the lower branch of dispersion curves; see Figure 7.

Lower branches of dispersion curves for $\mathrm{SH}$-waves in a two-layered traction-free plate

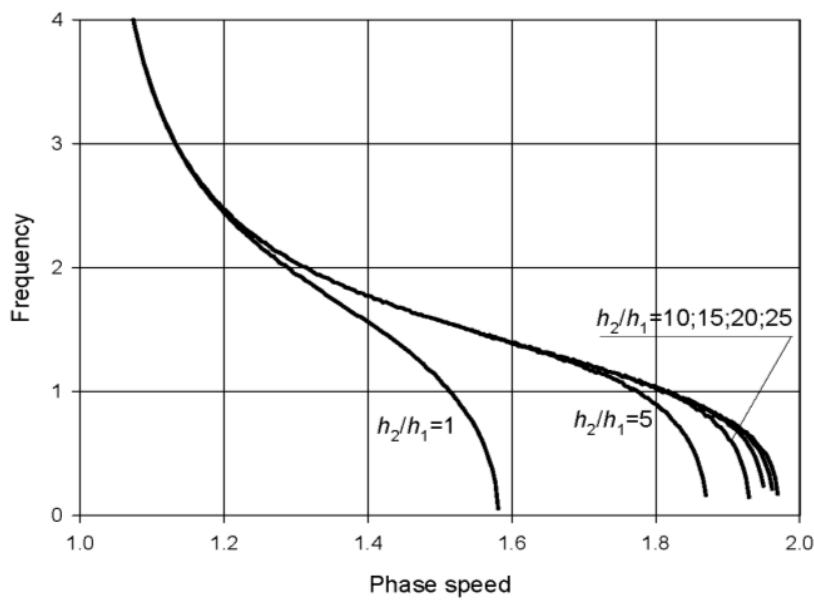

Figure 7. Lower branches of dispersion curves for SH-waves propagating in two-layered orthotropic plates with $\left(c_{\mathbf{n m}}^{T}\right)_{\min }=1$, $\left(c_{\mathbf{n m}}^{T}\right)_{\max }=2$, and different $h_{2} / h_{1}$ ratio

The presented data reveal that while ratio $h_{2} / h_{1}$ increases from 1 to 25 , the lower mode dispersion curves of the SH-wave are getting closer to the corresponding curve for Love wave; compare with Figure 5. However, even at the highest computed ratio $h_{2} / h_{1}=25$ there is a principle possibility to differentiate a $\mathrm{SH}$-wave from Love wave, since there limiting speeds remain distinguishable.

\section{Multilayered Orthotropic Plate}

Herein, we consider multilayered traction-free plates consisting of alternating layers (up to 31) of the unit thickness: $h_{n}=1, \quad n=1,2, \ldots, 31$. It is assumed that (i) all the layers are orthotropic with the axes of elastic symmetry coincident with vectors $\mathbf{n}, \boldsymbol{v}$, and $\mathbf{m}$; and (ii) the corresponding shear bulk waves of the contacting layers differ. Mechanical properties of the layers needed for the subsequent analysis are as follows:

$$
\left\{\begin{array}{l}
\left(c_{\mathbf{n m}}^{T}\right)_{2 k-1}=1 \\
\left(c_{\mathbf{n m}}^{T}\right)_{2 k}=2
\end{array} .\right.
$$

Since for a plate with more than 2 orthotropic layers no explicit analytical solution for the dispersion relations is known, a numerical algorithm exploiting the modified transfer matrix method will be adopted.

\subsection{MTM Method}

Applying the MTM method to a more general case of the $n$-layered plate with orthotropic layers of arbityrary thickness, we arrive at the implicit secular equation in the form [6]:

$$
\vec{T}^{(n)}\left(-h_{n} / 2\right) \cdot\left(\begin{array}{l}
\mathbf{M}_{n}^{-1}\left(h_{n} / 2\right) \\
\left(\prod_{k=2}^{n-1}\left(\begin{array}{l}
\mathbf{M}_{k}\left(-h_{k} / 2\right) \\
\cdot \mathbf{M}_{k}^{-1}\left(h_{k} / 2\right)
\end{array}\right)\right) \\
\cdot \mathbf{M}_{1}\left(-h_{1} / 2\right)
\end{array}\right) \cdot \vec{T}_{\perp}^{(1)}\left(h_{1} / 2\right)=0 .(7
$$

In Eq. (7.2) vectors $\vec{T}_{\perp}^{(1)}\left(h_{1} / 2\right)$ and $\vec{T}^{(n)}\left(-h_{n} / 2\right)$ represent the traction-free boundary conditions at outer planes, and $\mathbf{M}_{k}, \quad k=1, \ldots, n$ are the transfer matrices:

$$
\begin{aligned}
& {\overrightarrow{T_{\perp}}}^{(1)}\left(h_{1} / 2\right)=i r a_{1} \gamma_{1}\left(\begin{array}{c}
-\sinh \left(i r \gamma_{1} h_{1} / 2\right) ; \\
\cosh \left(i r \gamma_{1} h_{1} / 2\right)
\end{array}\right), \\
& \vec{T}^{(n)}\left(-h_{n} / 2\right)=i r a_{n} \gamma_{n}\left(\begin{array}{c}
\cosh \left(i r \gamma_{n} h_{n} / 2\right) ; \\
-\sinh \left(i r \gamma_{n} h_{n} / 2\right)
\end{array}\right), \\
& \mathbf{M}_{k}\left(x^{\prime}\right)=\left(\begin{array}{cc}
\sinh \left(i r \gamma_{k} x^{\prime}\right) & \cosh \left(i r \gamma_{k} x^{\prime}\right) \\
i r a_{k} \gamma_{k} \cosh \left(i r \gamma_{k} x^{\prime}\right) & i r a_{k} \gamma_{k} \sinh \left(i r \gamma_{k} x^{\prime}\right)
\end{array}\right),
\end{aligned}
$$

where $a_{k}=\mathbf{m} \otimes \mathbf{v} \cdot \mathbf{C}_{k} \cdot \boldsymbol{v} \otimes \mathbf{m}, \quad k=1, \ldots, n$.

Equation (7.2) is the transcendental equation with respect to the phase speed $c$ and wave number $r$. Applying relation (3.2), allows us to reformulate Eq. (7.2) in terms of the phase speed and frequency $\omega$. Direct evaluation of the determinant of the transfer matrix $\mathbf{M}_{k}$ reveals that $\operatorname{det} \mathbf{M}_{k}=-i r a_{k} \gamma_{k}$. Thus, the determinants do not vanish at $\gamma_{k} \neq 0$, and the transfer matrices in (7.2) are correctly defined at any (real and positive) phase speed satisfying the condition

$$
c \neq\left(c_{\mathbf{m n}}^{T}\right)_{1}, \ldots,\left(c_{\mathbf{m n}}^{T}\right)_{n} .
$$

Condition (7.4) is assumed to hold below.

REMARK 7.1. Expressions (7.3) correspond to orthotropic layers. When layers with monoclinic symmetry need to be considered, a more general expression accounting possible appearing of multiple roots in the Christoffel equation should be used; see $[5,6]$.

\subsection{Numerical Results}


Analysis of dispersion curves for SH-waves propagating in $n$-layered plates with different number of alternating layers (7.1) revealed that all the considered plates have similar lower branches ending at some finite phase speeds $c_{s}$, and the latter depend on number of layers; see Figure 8.

Lower branches of dispersion curves for $\mathrm{SH}$-waves in $n$-layered traction-free plates

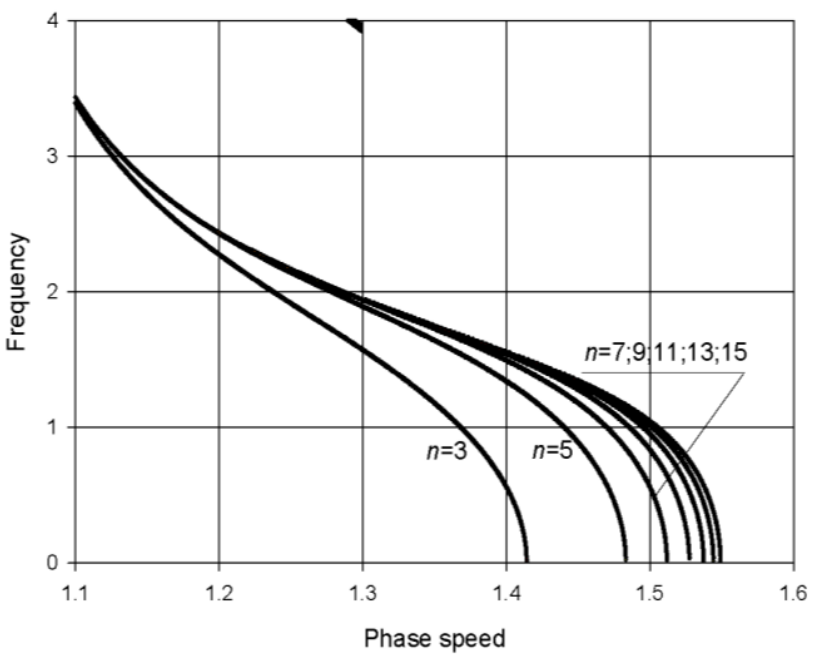

Figure 8. Lower mode dispersdion curves for the SH-waves propagating in $n$-layered plates

All these curves resemble one obtained for a twolayered plate; see Figure 6.

The following plot in Figure 9 demonstrates the first 50 dispersion curves in a 31-layered traction-free plate.

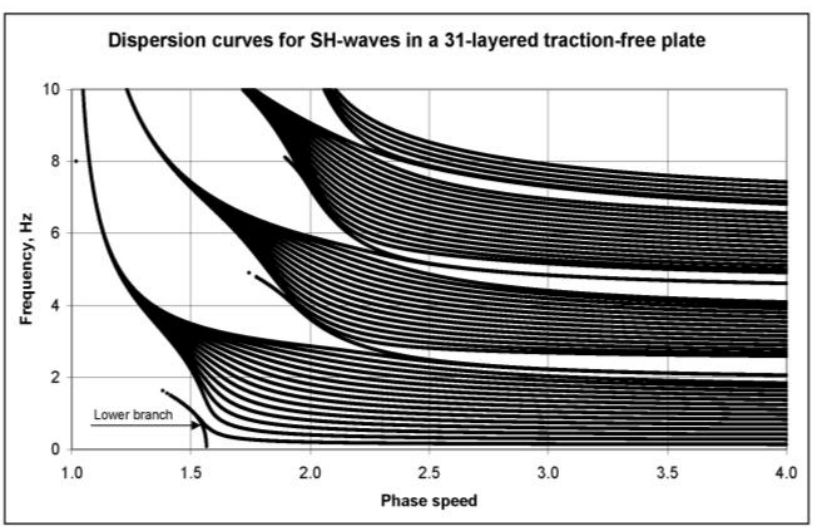

Figure 9. Dispersion curves for SH-waves in a traction-free 31-layered plate

The most interesting observable features in Figure 9 are: (i) existence of the anomalous wave speed $c_{s}$; (ii) fusion of the dispersion curves at their origin; and (iii) bundled structure of these curves.

REMARK 7.2. It should be noted that while properties (i) and (ii) are common for all the considered plates (7.1) at any $n \geq 2$, property (iii) is becoming stably observable at a relatively large number of layers $(n \geq 7)$.

Another plot in Figure 10 represents variation of the lower branch of the dispersion curves in a 31-layered traction-free plate due to the thickness variation of the 15th layer (that is the middle layer).
Variation of the lower branch in a 31-layered plate due to thickness variation of the middle layer

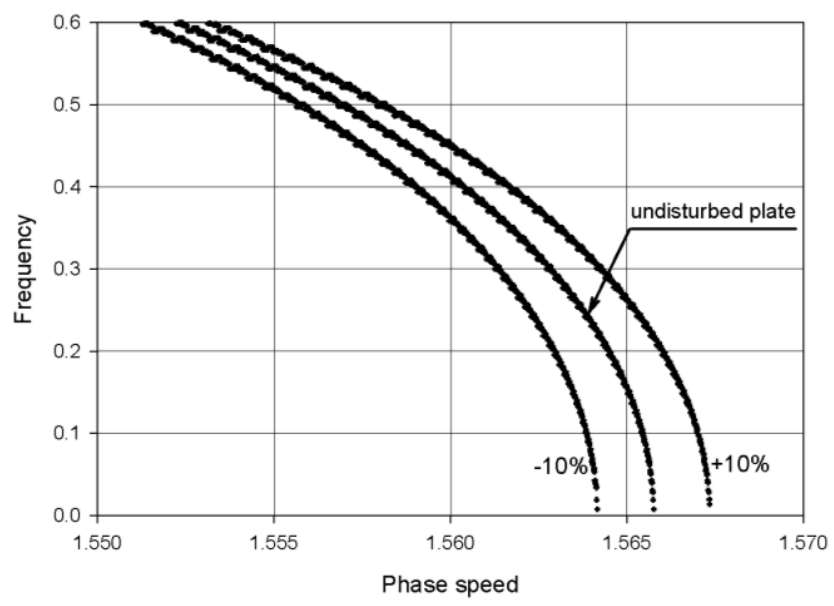

Figure 10. Variation of the lower branch in the vicinity of the speed $c_{S}$ due to $10 \%$ thickness variation of the middle layer in a traction-free 31 layered plate

These data shows that even relatively small $10 \%$ variation of the thickness of the middle layer is detectable by analyzing behavior of the lower branch in the vicinity of $c_{s}$. Similar results were obtained at variation of the shear bulk wave speed $\left(c_{\mathbf{n m}}^{T}\right)_{15}$ of the middle layer. Thus, analyzing lower branches of dispersion curves provides a robust and nondestructive method for evaluating physical and geometrical properties of the internal layers.

REMARK 7.3. To achieve the desired computational precision and avoid possible numerical instability, the main computations were implemented with mantissas having over 500 decimal digits. That was achieved by applying Bailey's numerical package for multiprecision computations.

\section{Acknowledgements}

Author thanks Dr. D.H. Bailey for the Multiprecision Package, the Russian Foundation for Basic Research (Grant 04-01-00781) and the Russian Academy of Sciences Program OEMMPU No.12 for partial financial support.

\section{References}

[1] A.E.H. Love, Some Problems of Geodynamics. Cambridge University Press, London (1911).

[2] E. Dieulesaint and D. Royer, Elastic Waves in Solids. Wiley, N.Y. (1980).

[3] T.C.T. Ting and D.M. Barnett, Classifications of surface waves in anisotropic elastic materials. Wave Motion 26 (1997) 207-218.

[4] S.V. Kuznetsov, Subsonic Lamb waves in anisotropic plates. Quart. Appl. Math. 60 (2002) 577-587.

[5] S.V. Kuznetsov, Love waves in stratified monoclinic media. Quart. Appl. Math. 62 (2004) 749-766.

[6] S.V. Kuznetsov, SH-waves in multilayered plates. Quart. Appl. Math. 64 (2006) 153-165.

[7] S.V. Kuznetsov, Love waves in non-destructive diagnostics of layered composites, Acoustical Physics, 56 (2010) 877-892.

[8] M.J.S. Lowe, Matrix techniques for modeling ultrasonic waves in multilayered media. IEEE Transactions on Ultrasonics, Ferroelectrics, and Frequency Control 42 (1995) 525-542. 
[9] D. Lévesque and L. Piché, A robust transfer matrix formulation for the ultrasonic response of multilayered absorbing media. $J$. Acoust. Soc. 92 (1992) 452-467.

[10] M. Castaings and B. Hosten, Transfer matrix of multilayered absorbing and anisotropic media. Measurments and simulations of ultrasonic wave propagation through composite materials. $J$. Acoust. Soc. Am. 94 (1993) 1488-1495.

[11] P. Michaels and V. Gottumukkula, Theory of viscoelastic Love waves and their potential application to near-surface sensing to permeability. In: Advances in near-surface seismology and ground-penetrating radar. Geophysical Developments Series. (2010) 263-278

[12] D. Restrepo, J.D. Gomez, and J.D. Jaramillo, SH wave number Green's function for a layered, elastic half-space. Part I: Theory and dynamic canyon response by the discrete wave number boundary element method. Pure Appl. Geophys. (2014) 1-14.

[13] M. Behm and R. Snieder, Love waves from local traffic noise interferometry. The Leading Edge. 32 (2013) 628-632.

[14] J. Xia, X. Yin, and Y. Xu, Feasibility of determining Q of nearsurface materials from Love waves. J. Appl. Geophysics. 95 (2013) $47-52$. 